\title{
Monitoring contractile dermal lymphatic activity following uniaxial mechanical loading
}

\author{
RJ Gray, PR Worsley, D Voegeli, DL Bader* \\ Clinical Academic Facility, Faculty of Health Sciences, University of Southampton, SO16 6YD Southampton, United Kingdom
}

\section{A R T I C L E I N F O}

\section{Article history:}

Received 23 December 2015

Revised 18 February 2016

Accepted 23 April 2016

Available online $\mathrm{xxx}$

\section{Keywords:}

Lymphatic system

Pressure ulcers

Fluorescence imaging

Objective quantification

\begin{abstract}
A B S T R A C T
It is proposed that direct mechanical loading can impair dermal lymphatic function, contributing to the causal pathway of pressure ulcers. The present study aims to investigate the effects of loading on human dermal lymphatic vessels.

Ten participants were recruited with ages ranging from 24 to 61 years. Participants had intradermal Indocyanine Green injections administrated between left finger digits. Fluorescence was imaged for 5 min sequences with an infra-red camera prior to lymph vessel loading, immediately after axial loading $(60 \mathrm{mmHg})$ and following a recovery period. Image processing was employed to defined transient lymph packets and compare lymph function between each test phase.

The results revealed that between 1-8 transient events (median $=4$ ) occurred at baseline, with a median velocity of $8.1 \mathrm{~mm} / \mathrm{sec}$ (range $4.1-20.1 \mathrm{~mm} / \mathrm{sec}$ ). Immediately post-loading, there was a significant $(p<0.05)$ reduction in velocity (median $=6.4$, range $2.2-13.5 \mathrm{~mm} / \mathrm{sec}$ ), although the number of transient lymph packages varied between participants. During the recovery period the number (range $1-7$ ) and velocity (recovery median $=9.6 \mathrm{~mm} / \mathrm{sec}$ ) of transient packets were largely restored to basal values.

The present study revealed that some individuals present with impaired dermal lymphatic function immediately after uniaxial mechanical loading. More research is needed to investigate the effects of pressure and shear on lymphatic vessel patency.
\end{abstract}

@ 2016 IPEM. Published by Elsevier Ltd. All rights reserved.

\section{Introduction}

The lymphatic system provides a transport mechanism responsible for fluid and protein homeostasis. In addition, it is responsible for supporting the immune system and the clearance of cell substances in the interstitium, thereby dissipating toxic products away from localised areas to ensure tissue health. Accordingly, where lymphatic clearance is impaired, these harmful substances will accumulate leading to cell death, tissue damage and a predisposition towards further pathology [1]. Although traditionally research has been focused on blood vessels, lymphatics have recently become popular in biomedical research, with its importance highlighted in cancer growth and metastasis [2].

There is a clinical need in the treatment and diagnosis of several medical conditions to delineate lymphatic morphology in a minimally invasive manner. For example, in the detection of sentinel lymph nodes during tumour resection or the identification of abnormal clearance patterns in the diagnosis of lymphoedema

\footnotetext{
Corresponding author. Tel.: +4402381204106.

E-mail address: D.L.Bader@soton.ac.uk (D. Bader).
}

[3]. This has traditionally been achieved through injection of a radioisotope, typically Technetium-99 $\mathrm{m}$, and subsequently tracking the gamma radiation, in a process termed lymphoscintigraphy [4]. However, in recent years, near-infrared (NIR) imaging techniques have emerged as a feasible alternative to replace these radiological approaches [5]. This NIR system offers the additional benefits of minimal absorption and scattering of light by tissue components within its optical window, corresponding to the excitation of fluophores, which are appropriate for imaging structures within biological tissues. Indeed, fluorescent agents, such as Indocyanine Green (ICG, CardioGreen), in conjunction with the NIR imaging, have proved as effective in delineating lymphatic vessels when compared to contrast dyes and radioisotopes [6].

Recent research has implicated impaired lymphatic clearance as one of the mechanisms associated with the aetiology of pressure ulcers [7]. This is supported by a seminal experimental study using lymphoscintigraphy with a canine model, in which Miller and Seale [8] reported impaired lymphatic clearance at a critical uniaxial pressure between $60 \mathrm{mmHg}(8 \mathrm{kPa})$ and $75 \mathrm{mmHg}(10 \mathrm{kPa})$. In a subsequent study, these authors demonstrated that recovery of lymphatic clearance was found to be highly dependent on the 
A

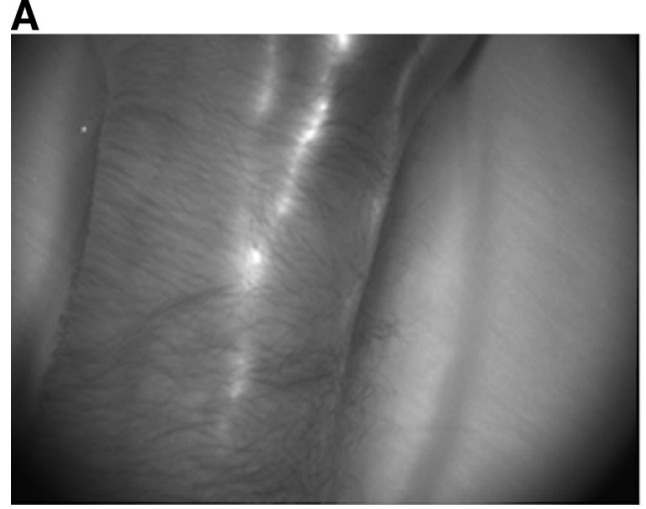

B

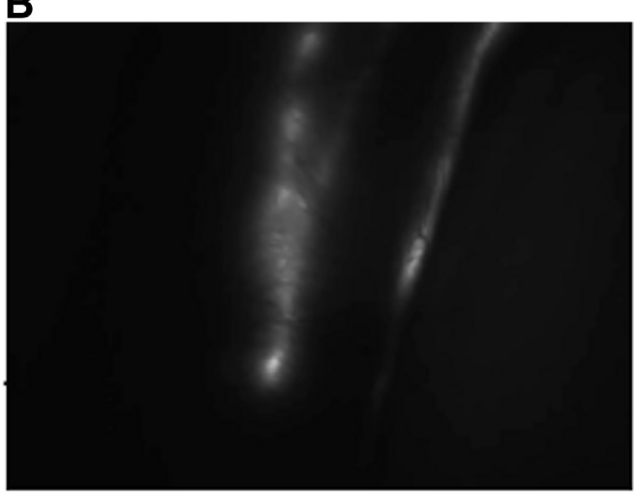

Fig. 1. (a) Participant forearm following ICG injection, (b) successful delineation of dermal lymphatic vessels.

magnitude of the post-occlusive pressure [9]. Adopting a similar approach with radioisotopes is contraindicated in healthy human volunteers due the inevitable risks associated with radiation exposure to the participants.

To date, related research on humans has been restricted to subjective measures of lymph flow using lymphography techniques with cuffs or restrictive collars [10]. The authors of the present study have recently adopted a minimally invasive approach, using Indocyanine green (ICG) fluorescence imaged with an NIR camera, to qualitatively describe the effects of applied uniaxial loading on lymph vessel patency [11]. However, there is a need to provide objective quantification of lymphatic vessel function before and after mechanical loading to provide a basis for reliable clinical translation. Accordingly the present study was designed to quantify peripheral dermal lymphatic function pre- and post-uniaxial mechanical loading using ICG fluorescence imaging techniques. This involved developing an analytical methodology to estimate robust parameters which reflect the dynamic behaviour of transient lymphatic packets.

\section{Materials and methods}

\subsection{Participants}

Ten participants ( 3 female and 7 male) were recruited into the study with ages ranging between 24 and 61 years. Exclusion criteria included a history of skin damage or disease and contraindications for Cardiogreen injections. All participants were provided with full details of the study prior to giving informed consent. Ethics Approval for the study was granted by the Local Institutional Committee at the University of Southampton (REC ID: 8209).

\subsection{Methodology}

Participants were seated with their left arm supported on a vacuum consolidation pillow at the level of the heart. They were requested to remain as still as possible for the duration of the study, to minimize the contribution of passive mechanisms to lymphatic clearance and reduce the impact of movement artefact during NIR imaging. Indocyanine green (ICG) injections of $50 \mu \mathrm{L} 0.05 \% \mathrm{w} / \mathrm{v}$ at a shallow intradermal depth were administrated to delineate dermal vessels [11]. Single injections were delivered into the two inter-digital spaces between the thumb and the second finger by a registered nurse (DV), constituting a total micro-dose of $0.05 \mathrm{mg}$. To encourage rapid uptake of ICG into lymphatic vessels, participants were instructed to clench their fists 20 times following injection. Upon successful ante-cubital delineation of lymphatic vessels (Fig. 1), participants were requested to relax for a further 5 mins, during which a suitable focal point for loading on an active dermal vessel was identified.

The focal point for loading on the volar aspect of the wrist was selected based on the following criteria; (i) A well-imaged area, suitable for loading without causing discomfort to the participant (ii) The vessel displaying frequent contractile activity resulting in transient lymph packets (iii) Post-summation and post-divergence of vessels. A specially designed indenter, with nominal diameter of $42.5 \mathrm{~mm}$ and curved edges to minimise stress concentrations, was mounted on a cantilever beam with an incorporated load cell [11]. A load was prescribed equivalent to a pressure of $60 \mathrm{mmHg}(8 \mathrm{kPa})$, for a period of 40 mins (Fig. 2).

Excitation of ICG and video capture of fluorescence emission was achieved using an NIR imaging system (Fluobeam ${ }^{\circledR}$ 800) with associated software (Fluobeam v3.1.1, Fluopotics, France). The system incorporates an integrated laser $(780 \mathrm{~nm})$ and CCD sensor, with appropriate filters to isolate fluorescence of ICG (peak $830 \mathrm{~nm}$ ). Three videos, each 5 mins in duration, were captured at three time points, namely, pre-load (T0), immediately after a 40 min period of axial loading (T40) and after a 40 min recovery period (T80). Frame acquisition was captured at frequencies of between 3 and $4 \mathrm{~Hz}$, individually optimised to maximise detection of vessels and minimise greyscale saturation.

\subsection{Video analysis}

In order to track and quantify transient lymph activity a droplet morphometry and velocimetry (DMV) technique was applied [12]. This iterative technique provided a selection of parameters which reflected lymphatic function, namely, the relative spatial and time dependent properties of transient lymph packets. Each transient event was analysed to provide $x$ and $y$ coordinates of the packet centroids, whereby the resultant displacements could be estimated (Supplementary Video). In addition, for each packet the mean area, velocity and intensity were calculated. Analysis was conducted offline using custom software (Matlab, Mathworks, USA) in five distinct phases to determine the robust parameters from the lymphography videos. Fig. 3 highlights the points at which sensitivity analyses were conducted during the image processing procedure and the resultant tracking of lymph packets (bottom of figure).

\subsubsection{Background image subtraction}

Background subtraction was used to differentiate transient lymphatic packets from other features in the video image. A sensitivity analysis of three different subtraction methods was undertaken on 150 frames of videos selected at random for each participant. The three methodologies involved: 


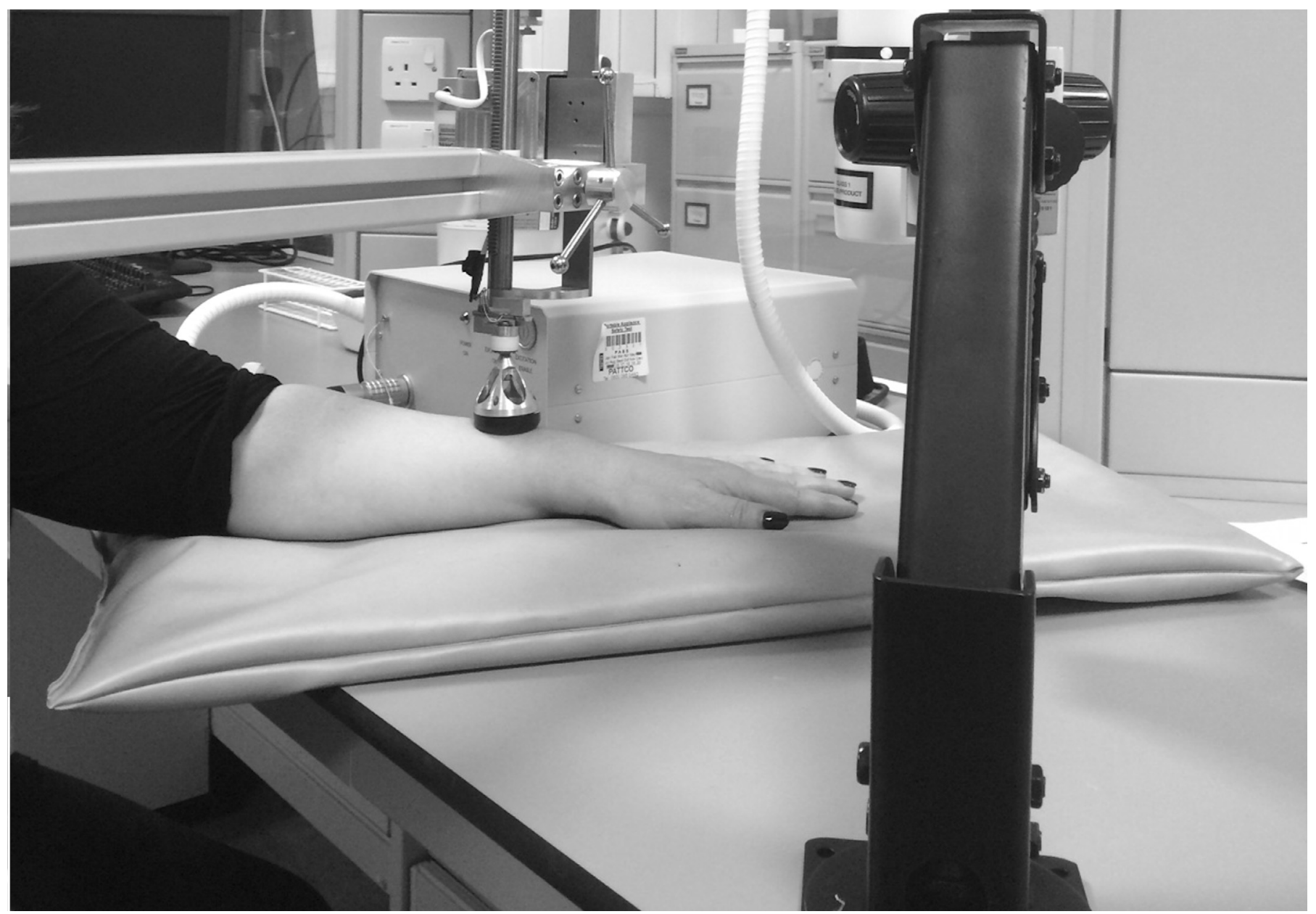

Fig. 2. Example mechanical loading over the volar aspect of a participant's wrist.

1. Subtraction of the first frame $\left(F_{n}-F_{1}\right)$

2. Subtraction of the previous frame $\left(F_{n}-F_{n-1}\right)$

3. Subtraction of the moving average of 10 previous frame $\left(F_{n}-\right.$ $\left.\frac{F_{n-1,2 \ldots .10}}{10}\right)$

Following each method the resultant peak intensities were plotted for the 150 frame sequences. The performance of the subtraction method was evaluated by estimating the root mean squared (RMS) value of the signal and identifying areas of peak intensity which exceeded three times this RMS value (Fig. 4).

\subsubsection{Binary conversion}

The greyscale image sequences were converted to binary using an intensity threshold. This threshold was selected following sensitivity analysis, whereby a range of values were iteratively assessed above and below a perceived optimum. This perceived optimal value was identified by assessing the maximum pixel intensity for each frame across a given video following the moving average subtraction method. Here a threshold was selected as the point in which transient packages were retained while discarding the background noise (Fig. 5). Iterations of three intensity points above and below the perceived optimum were selected to provide the analysis resulting in an intensity range of \pm 21 points. In order to analyse the effects of the threshold the number of transient packets in each binary video sequence was calculated and their frequencies were cross-referenced with the original data capture to assess for sensitivity and specificity. The threshold which optimized both these measures was selected for subsequent analysis.

\subsubsection{Small object removal and shape discrimination}

In order to filter noise or erroneous objects created in the binary conversion, two distinct techniques were employed; (i) contiguous objects with an area smaller than $3 \mathrm{~mm}^{2}$ were removed, (ii) shape discrimination was applied to ensure only transient events were detected using a circle metric defined as $>50$ connecting pixels.

\subsubsection{Lymph packet tracking}

Unique transient lymph packets were subsequently tracked over multiple frames, to calculate the trajectory, velocity, shape, and other time-dependent parameters. In order to provide this tracking capability an extended Kalman filter (EKF) was applied to ensure centroid axes were linked between video frames. This resulted in a smooth profile of centroid movement of each packet over multiple video frames.

\subsubsection{Post-processing and data analysis}

The above steps were repeated for each frame of a video to produce a time history database of each unique transient lymph packet. For each packet a number of parameters were estimated, including $x$ displacement from lymph node, $y$ displacement from lymph node, the average velocity, the average area of packets and the average pixel intensity.

\subsection{Statistical analysis}

Participant data sets were presented to describe transient lymph behaviour across each of the test phases, namely, T0, 


\section{Data Capture}

.avi video $900-1000$ frames at $4-7 \mathrm{~Hz}$
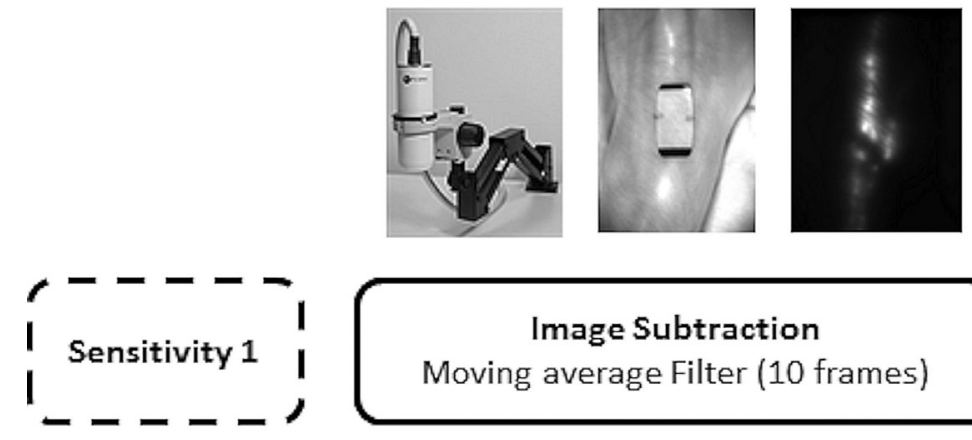

Image Subtraction

Moving average Filter (10 frames)
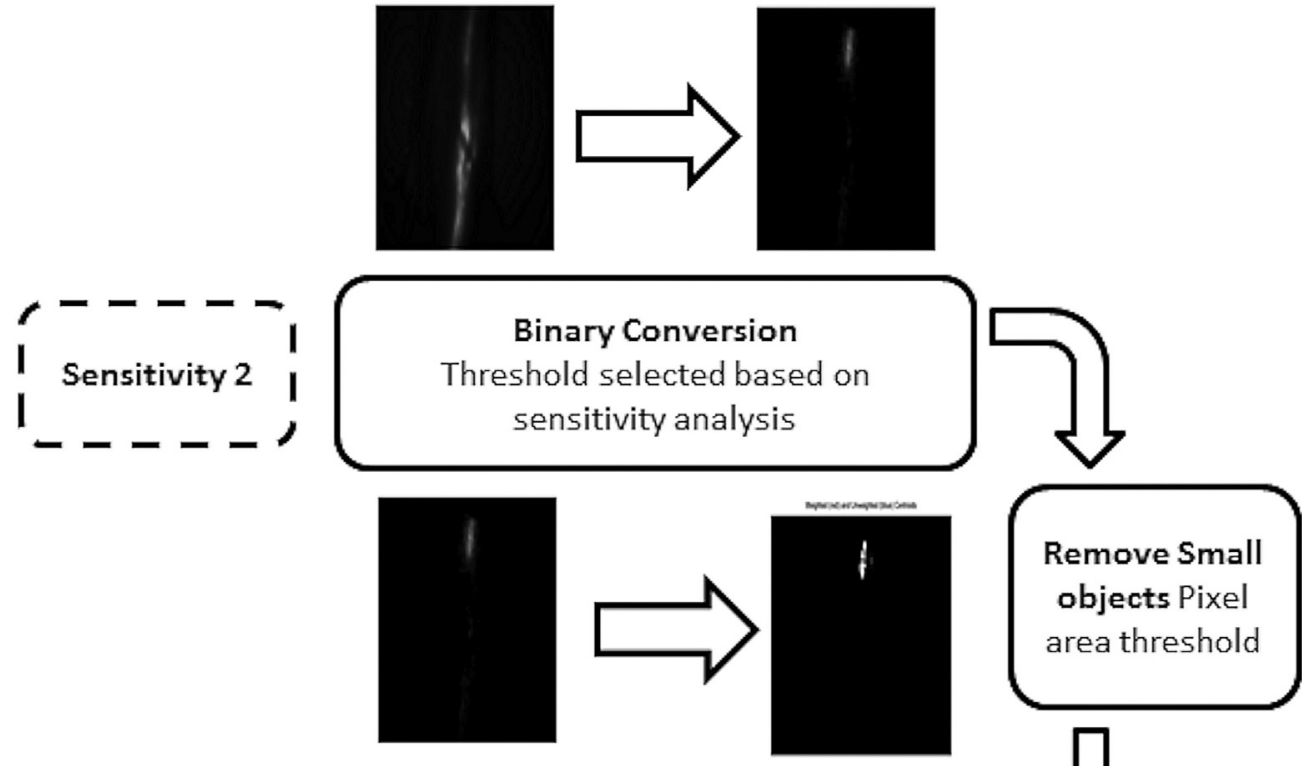

\section{Lymph Packet Tracking}

track centroid of binary image using

Extended Kalman Filter (EKF)
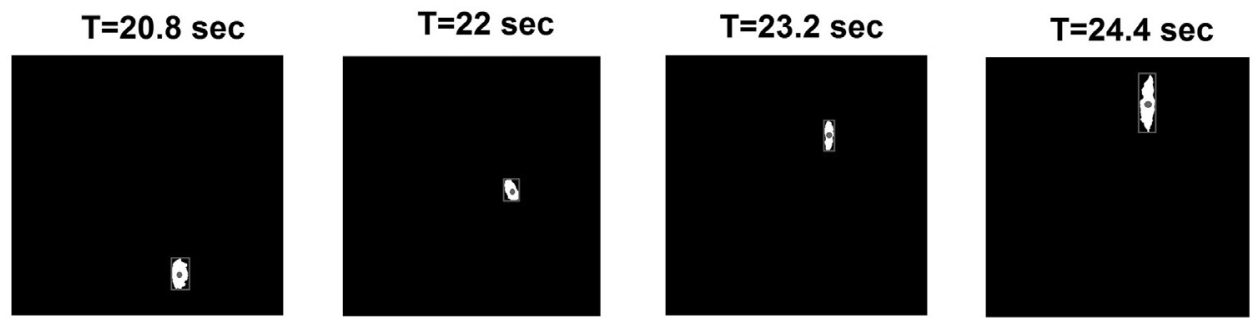

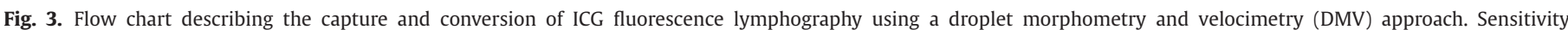

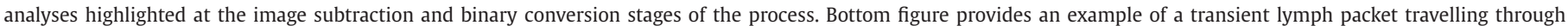
a dermal lymphatic vessel over a $2.6 \mathrm{~s}$ period.

T40 and T80. Data were also collated from all participants and presented in non-parametric descriptors (median). Subsequent analysis of changes between T0, T40 and T80 were conducted using a Friedman test, using a 95\% confidence interval. Comparisons between post-loading data (T40 and T80) and baseline values were conducted using Wilcoxon signed-rank tests. In addition, comparisons between collection periods were made between participants who exhibited visual pathological changes in lymphatic valve function (backflow events) and those whose lymph function remained similar across each of the test phases. This was achieved by defining trends in lymphatic function using categorical analysis. 


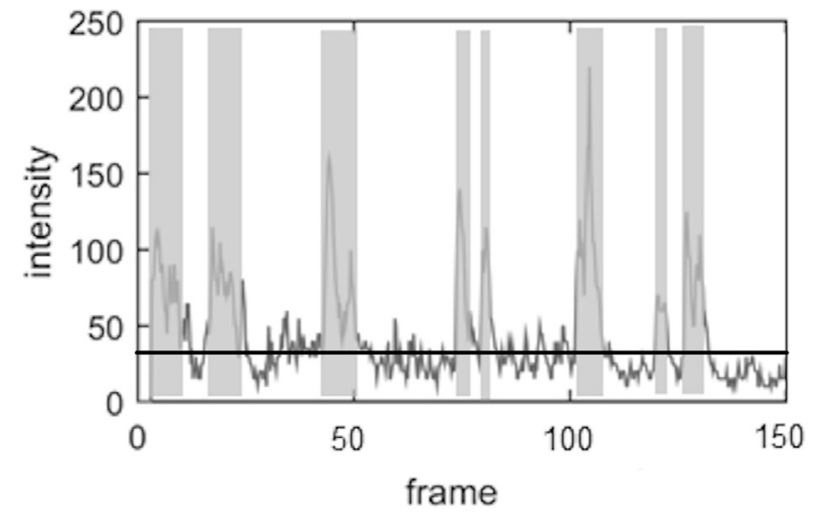

Fig. 4. Peak intensity over 150 frames of a video sequence, with the shaded area representing an intensity greater than three times the root mean squared (RMS) line value as indicated.

\section{Results}

\subsection{Sensitivity analysis}

Sensitivity studies were conducted to evaluate the effects of the image subtraction methodology and the threshold selection for binary conversion.

\subsubsection{Image subtraction}

The results revealed that when the subtraction methods were compared the moving average technique was the most efficient in removing background noise whilst providing detail of transient lymph packets. For this method $100 \%$ of the observed transient events were detected in the peak intensity plot, with the profile providing large changes in maximum intensity during transient packet events (large deviation from the RMS signal). By contrast, the first frame and previous frame subtraction methods only detected $30 \%$ and $60 \%$ of the transient events respectively. This can be illustrated in Fig. 6, where, for example, the first frame subtraction method failed to discriminate packets based on peak frame intensity (Fig. 6a). By contrast the moving average method produced a significant improvement in signal noise ratio (Fig. 6c) and was applied for the subsequent video analyses.

\subsubsection{Intensity threshold selection}

The analyses revealed that with low thresholds there was a high sensitivity of detecting transient lymph activity, associated with a poor specificity (Fig. 7). As the threshold increases towards the perceived optimum the specificity increased to $58 \%$ associated with a sensitivity of $81 \%$. For subsequent increases of the threshold intensity the sensitivity continued to decrease, with only marginal increases in specificity.

\subsection{Baseline lymphatic function (TO)}

Episodes of transient lymph packets were observed in all baseline (T0) videos (Table 1). The lymphatic vessels were orientated in the cephlad (towards the head) direction resulting in significant transient displacements in the $Y$ direction (range $14-74 \mathrm{~mm}$ ). The analyses revealed there were between 1-8 transient events (median $=4)$, with an average velocity ranging between 4.1 and $20.1 \mathrm{~mm} / \mathrm{s}$ (median $=8.1 \mathrm{~mm} / \mathrm{sec}$ ). There was some variability in the number and speed of transient packets across a single data collection session. However, the trend in movement pattern was consistent within participants.

\subsection{Immediately post-loading (T40)}

Immediately following mechanical loading, video analysis suggested evidence of damage to vessel and valves, in the form of backflow events, in four participants (Table 2). There were different trends in the number of transient lymphatic packages when compared to baseline data. Thus four participants demonstrated a similar number (P4, P5, P6 and P7), three participants a reduced number (P2, P9 and P10), while in three participants there was an increase in the number of transient packets (P1, P3 and P8). The reduction in velocity of transient packets (median $=6.4$, range $2.2-13.5 \mathrm{~mm} / \mathrm{s})$ was statistically significant $(p<0.05)$ when compared to $T 0$ velocity values. However, differences in the magnitude of travel, area of packets and intensity of packets were all not statistically significant $(p>0.05)$.

\subsection{Recovery period (T80)}

Analysis of the recovery period video revealed that $2 / 3$ vessels which presented with vessel damage had recovered, with only P2 still demonstrating some backflow at T80 (Table 3). The number of transient events ranged from 1 to 7 , with the median velocity of transient packets of $9.6 \mathrm{~mm} / \mathrm{s}$. The magnitude of travel followed a similar pattern to that described for each participant in $\mathrm{T} 0$ and T40 conditions, ranging from 16.9 to $82.2 \mathrm{~mm}$. Statistical analyses revealed that none of the parameters in the recovery period (T80) were significantly different $(p>0.05)$ to that of baseline data (T0).

When comparisons were made between combined test conditions (T0, T40 and T80) there were no significant differences $(p>0.05)$ in packet numbers, displacement (Fig. 8), area or intensity of transient lymph packets. However, there was a significant difference in velocity $(p<0.05)$, with an associated reduction in transient packet velocity immediately post-loading (T40

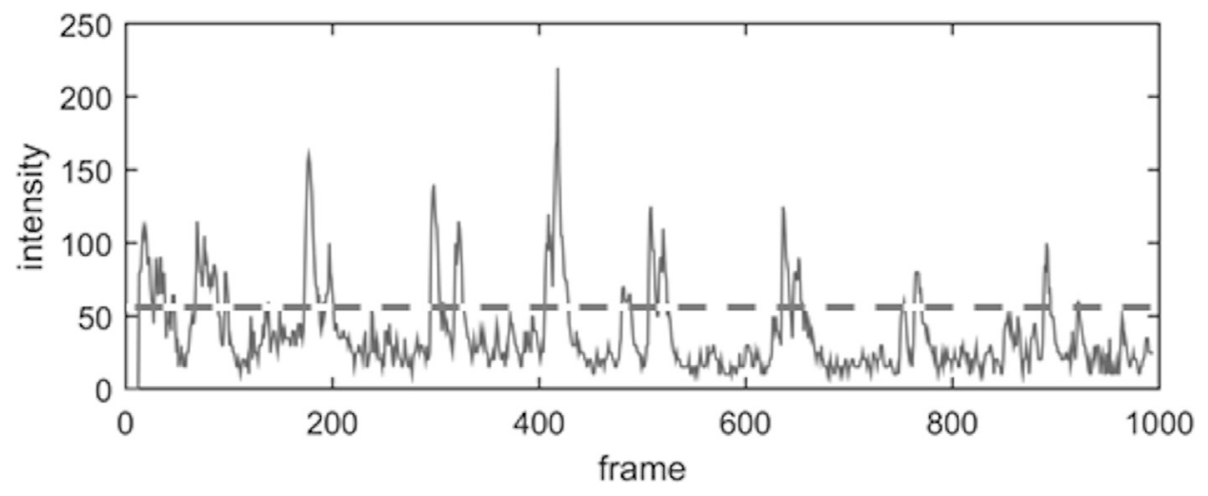

Fig. 5. Peak intensity profile for one video sequence with perceived optimal threshold highlighted (dotted line). 
a
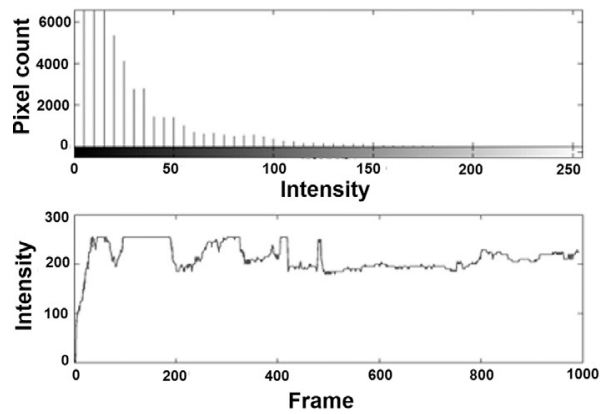

b
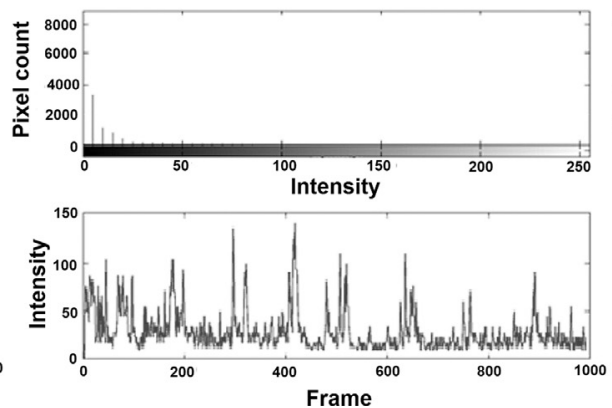

C
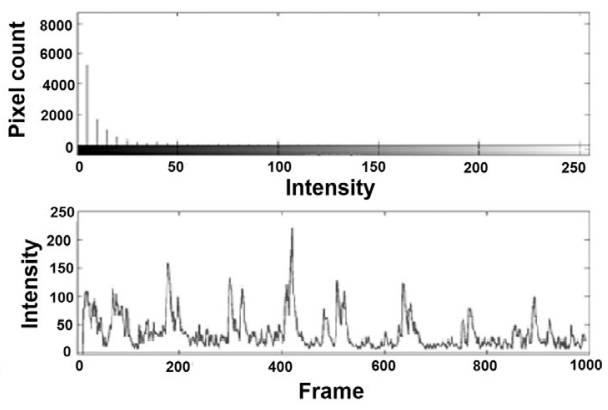

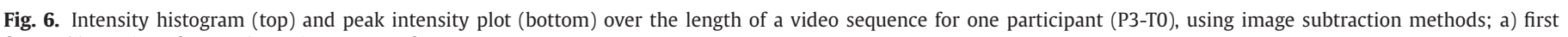
frame, b) previous frame, c) moving average frame.

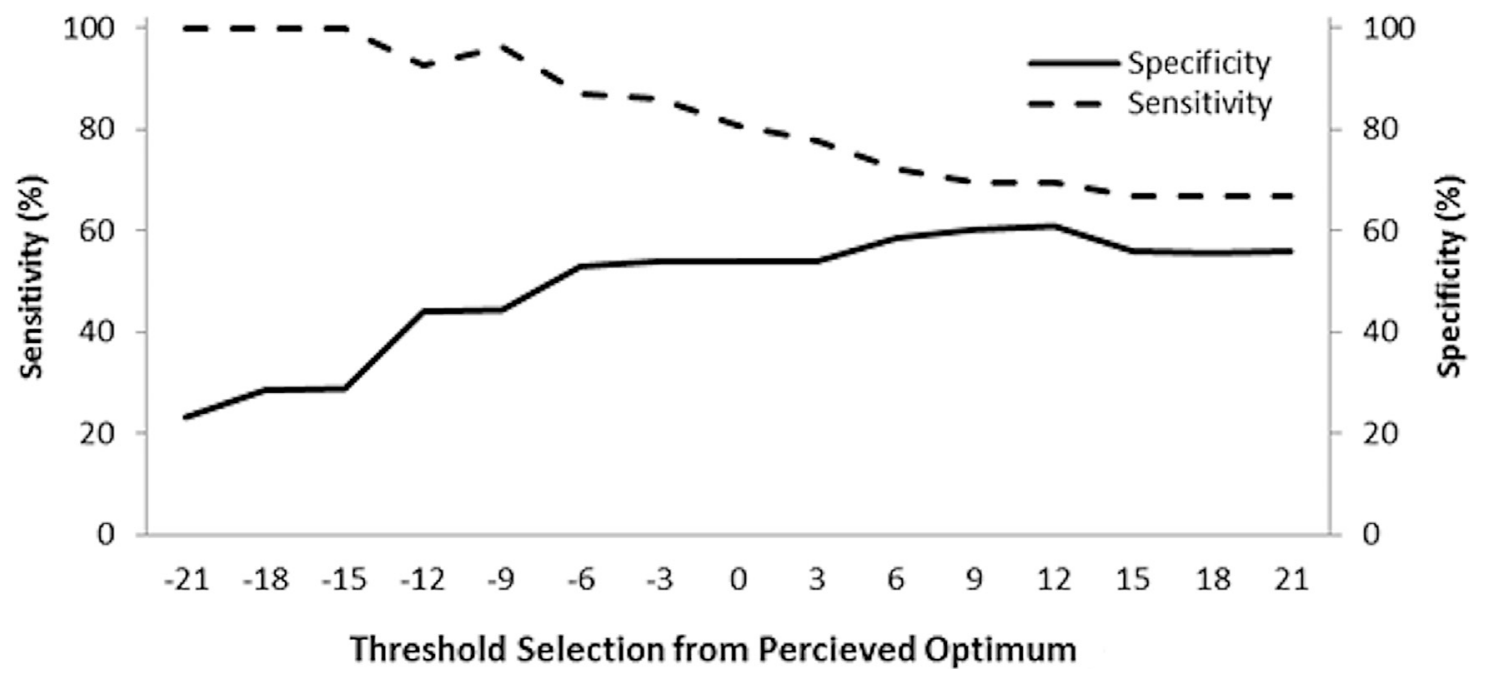

Fig. 7. Mean sensitivity and specificity of the intensity threshold to detect transient lymphatic activity.

median $=6.4$, range $2.2-13.5 \mathrm{~mm} / \mathrm{s}$ ) compared to baseline (T0 median $=8.1 \mathrm{~mm} / \mathrm{s})$ and recovery $($ T80 median $=9.6 \mathrm{~mm} / \mathrm{s})$ phases.

\subsection{Categorical trends}

When observing trends across the three temporal phases of data collection, the response of the lymphatic vessels to mechanical loading could be divided into three categories (Fig. 9);
1. Normal Function-a similar number of transient lymphatic packages were observed over the data capture period for each phase of the test protocol (P4, P5 and P7).

2. Increased activity post-loading-transient lymphatic packets increased immediately post-loading (T40) when compared to baseline (T40). However, following the recovery phase (T80), the number of transient packages reduced again (P1, P3, P6 and P8)

3. Decreased activity post-loading-transient lymphatic packet numbers decrease immediately post-loading (T40). Function

Table 1

Summary of parameters estimated from baseline unloaded data (T0).

\begin{tabular}{|c|c|c|c|c|c|c|c|}
\hline \multirow[b]{2}{*}{ Participant } & \multirow[b]{2}{*}{ No of packets } & \multicolumn{2}{|c|}{ Peak travel (mm) } & \multirow[b]{2}{*}{ Evidence of damage } & \multirow[b]{2}{*}{ Velocity (mm/s) } & \multicolumn{2}{|c|}{ Average packet attributes } \\
\hline & & $X$ & $Y$ & & & Area & Intensity \\
\hline 1 & 2 & 3.0 & 74.0 & NA & 20.1 & 1699 & 174 \\
\hline 2 & 8 & 4.7 & 26.3 & NA & 7.1 & 1383 & 87 \\
\hline 3 & 6 & 17.5 & 27.3 & NA & 12.6 & 1255 & 162 \\
\hline 4 & 3 & 19.4 & 33.8 & NA & 7.6 & 2836 & 198 \\
\hline 5 & 2 & 27.3 & 32.5 & NA & 4.1 & 1483 & 175 \\
\hline 6 & 4 & 3.8 & 28.9 & NA & 7.5 & 1402 & 193 \\
\hline 7 & 1 & 3.6 & 14.3 & NA & 8.6 & 1061 & 221 \\
\hline 8 & 3 & 7.9 & 33.3 & NA & 7.2 & 3634 & 57 \\
\hline 9 & 5 & 34.2 & 37.6 & NA & 15.8 & 2144 & 226 \\
\hline 10 & 5 & 15.0 & 51.7 & NA & 9.1 & 1115 & 195 \\
\hline Median & 3.5 & 11.45 & 32.9 & & 8.1 & 1442.5 & 184.2 \\
\hline Range & $1-8$ & $3.0-34.2$ & $14.3-74.0$ & & $4.1-20.1$ & $1061-3634$ & $57-226$ \\
\hline
\end{tabular}


Table 2

Summary of parameters estimated from data capture videos immediately post-loading (T40).

\begin{tabular}{|c|c|c|c|c|c|c|c|}
\hline \multirow[b]{2}{*}{ Participant } & \multirow[b]{2}{*}{ No of transient packets } & \multicolumn{2}{|c|}{ Peak travel (mm) } & \multirow[b]{2}{*}{ Evidence of damage } & \multicolumn{3}{|c|}{ Average packet attributes } \\
\hline & & $X$ & Y & & Velocity (mm/sec) & Area & Intensity \\
\hline 1 & 6 & 7.5 & 53.8 & NA & 13.5 & 1023 & 192 \\
\hline 2 & 3 & 11.3 & 14.1 & Backflow & 2.2 & 2462 & 226 \\
\hline 3 & 8 & 38.5 & 54.0 & NA & 10.3 & 1250 & 208.0 \\
\hline 4 & 4 & 15.4 & 26.3 & Backflow & 11.7 & 1607 & 186 \\
\hline 5 & 2 & 6.4 & 32.9 & Backflow & 2.9 & 3634 & 57 \\
\hline 6 & 6 & 8.0 & 52.6 & NA & 5.3 & 1642 & 164 \\
\hline 7 & 1 & 5.1 & 50.4 & Backflow & 7.2 & 1784 & 185 \\
\hline 8 & 7 & 3.9 & 38.0 & NA & 7.7 & 3026 & 215 \\
\hline 9 & 5 & 16.9 & 45.1 & NA & 4.8 & 1260 & 187 \\
\hline 10 & 3 & 21.2 & 50.6 & NA & 5.6 & 1995 & 212 \\
\hline Median & 4.5 & 9.7 & 47.8 & & 6.4 & 1713 & 190 \\
\hline Range & $1-8$ & $3.9-38.5$ & $14.1-54.0$ & & $2.2-13.5$ & $1021-3634$ & $57-226$ \\
\hline
\end{tabular}

Table 3

Summary of parameters estimated from data capture videos during the recovery phase of testing (T80).

\begin{tabular}{|c|c|c|c|c|c|c|c|}
\hline \multirow[b]{2}{*}{ Participant } & \multirow[b]{2}{*}{ No of transient packets } & \multicolumn{2}{|c|}{ Magnitude of travel (mm) } & \multirow[b]{2}{*}{ Evidence of damage } & \multirow[b]{2}{*}{ Av velocity (mm/sec) } & \multicolumn{2}{|c|}{ Average packet attributes } \\
\hline & & $\mathrm{X}$ & $\mathrm{Y}$ & & & Area & Mean intensity \\
\hline 1 & 5 & 11.3 & 47.2 & NA & 10.1 & 937 & 163 \\
\hline 2 & 1 & 23.7 & 47.0 & Backflow & 3.9 & 1557 & 214 \\
\hline 3 & 2 & 9.4 & 31.4 & NA & 10.9 & 1012 & 221 \\
\hline 4 & 5 & 18.8 & 35.7 & NA & 12.8 & 3927 & 210 \\
\hline 5 & 2 & 11.7 & 32.9 & NA & 3.8 & 1139 & 182 \\
\hline 6 & 3 & 2.6 & 82.2 & NA & 8.1 & 1906 & 218 \\
\hline 7 & 3 & 7.3 & 48.5 & NA & 11.5 & 1635 & 203 \\
\hline 8 & 5 & 7.5 & 60.0 & NA & 9.1 & 3027 & 204 \\
\hline 9 & 7 & 15.0 & 62.0 & NA & 10.1 & 2487 & 155 \\
\hline 10 & 1 & 7.7 & 16.9 & NA & 8.2 & 1187 & 188 \\
\hline Median & 3 & 10.4 & 47.0 & & 9.6 & 1596 & 203 \\
\hline Range & $1-7$ & $2.6-23.7$ & $16.9-82.2$ & & $3.8-12.8$ & 937-3927 & $155-221$ \\
\hline
\end{tabular}

may or may not return during the recovery phase (T80) of the protocol (P2, P9, P10)

\section{Discussion}

Based on the recent study by the authors which involved qualitative assessment of loaded human lymphatic vessels [11], the present work derives a series of robust quantitative parameters to define lymphatic function both prior to and following the application of uniaxial mechanical loading. A sensitivity analysis was performed to verify the image processing techniques which were used to define transient lymphatic activity. This resulted in an optimised image processing technique that was capable of isolating moving transient lymphatic packets and quantifying their features including number, displacement, velocity and size. The proposed techniques could also provide insight for investigating when lymph nodes become blocked by infection, tumour metastasis, or surgical removal. The analytical technique has already shown potential for aiding the diagnosis of common lymphatic pathology [13].

The results of the study have demonstrated that an applied pressure of $60 \mathrm{mmHg}$ ( $8 \mathrm{kPa}$ ) can compromise dermal lymphatic vessels resulting in impaired valve function and backflow events in some of the able-bodied participants. These findings are in concert with the previous animal model involving radioisotope tracers in which lymphatic clearance was impaired at critical uniaxial pressures of between $60 \mathrm{mmHg}(8 \mathrm{kPa})$ and $75 \mathrm{mmHg}(10 \mathrm{kPa})$ [8]. The duration of loading was similar for that of Miller and Seale (30 mins) and the present study (40 mins). Further investigation is required to establish whether load duration as well as magnitude plays a role in lymphatic impairment. Direct comparisons between the present study and previous work on animal models must be treated with caution as the studies used different imaging protocols and there are differences in lymphatic anatomy and physiol- ogy between species. To date, related research on humans has been restricted to subjective measures of lymph flow using lymphography techniques involving ICG with cuffs or restrictive collars [10]. There is clearly a need for future research to assess post-occlusive pressures on human dermal lymphatic vessels.

Three trends were observed in the behaviour of lymphatic function following mechanical loading (Fig. 9). The authors hypothesize causal factors in underpinning these three trends. Participants with minimal changes in transient activity were relatively unaffected by the period of uniaxial loading and the dermal lymphatic vessels/valves remained functional throughout the three test phases (T0, T40 and T80). In those participants where the transient activity increased, it suggests that during the loading phase vessels may have been occluded and removal of the pressure induced increased activity within the lymphatic vessel. By contrast, direct damage to vessels and/or valves probably occurred in those participants who exhibited decreased activity following loading. Interestingly for the participant (P2) who had sustained evidence of backflow (both T40 and T80) there was a continuous reduction in the number of transient packets over the three imaging phases $(\mathrm{T} 0=8$, $\mathrm{T} 40=3, \mathrm{~T} 80=1)$.

Limitations of the present study include the sample size $(n=10)$ and sensitivity of the DMV technique to define lymph activity, particularly during the selection of the binary conversion threshold. This potential error, however, was minimized by the use of sensitivity analyses, although it is clear that selection of the binary threshold value has a substantive effect on the resultant detection of transient packets (Fig. 7). The present study assessed lymph vessel function immediately following loading (T40), with no analysis of vessel patency during the loading period. NIR imaging during loading would require some alteration to the test system and could include the application of both direct pressure and shear forces, which are implicated in the development of 


\section{Baseline T0}

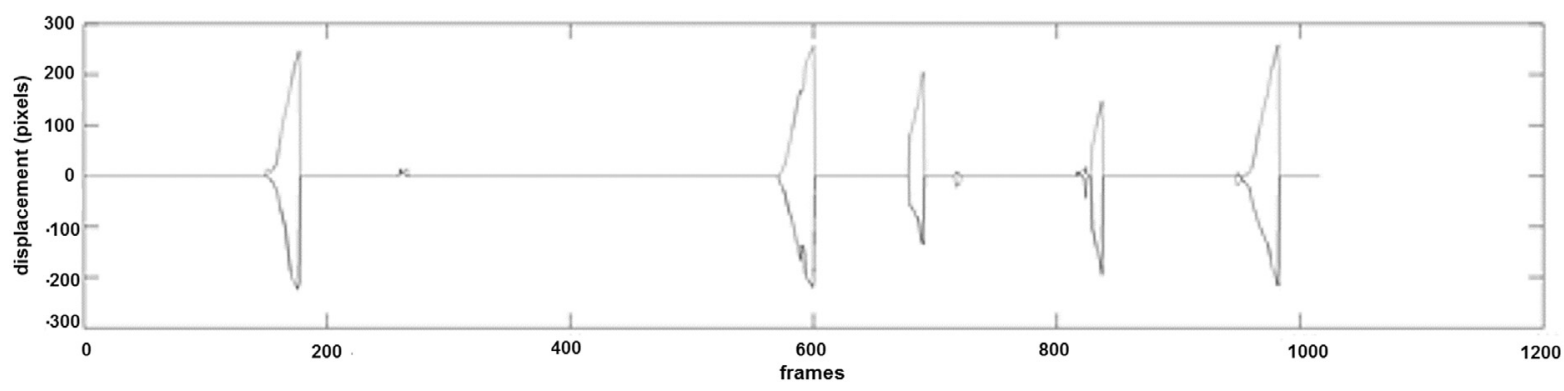

Post-loading T40

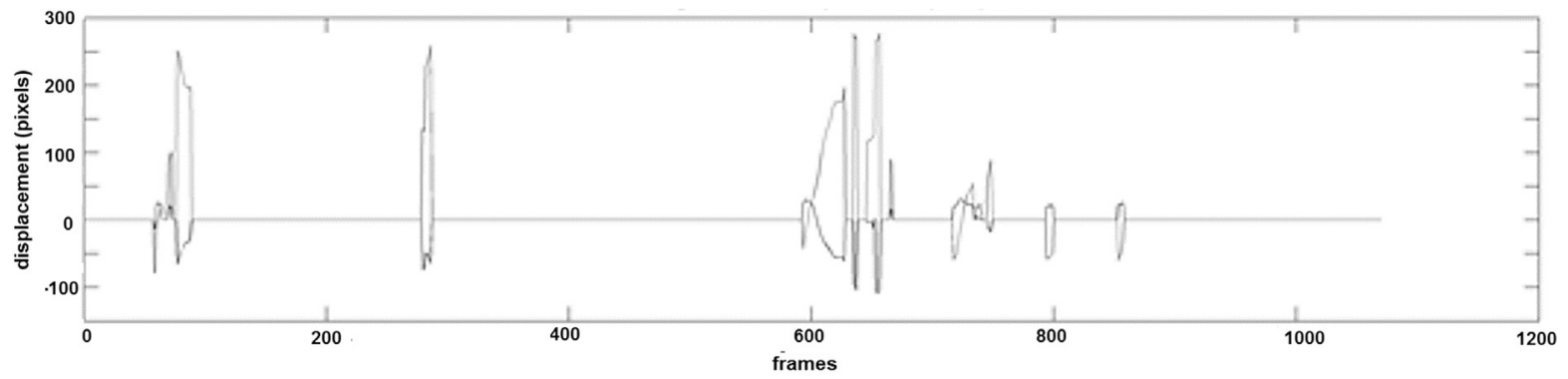

Recovery period T80

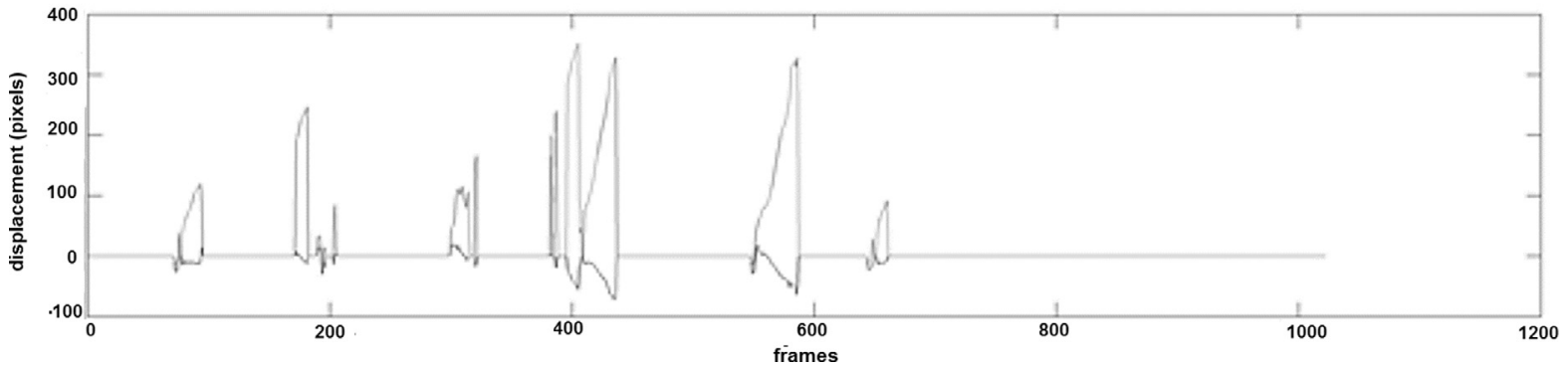

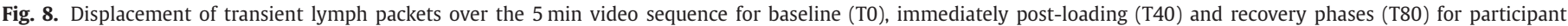

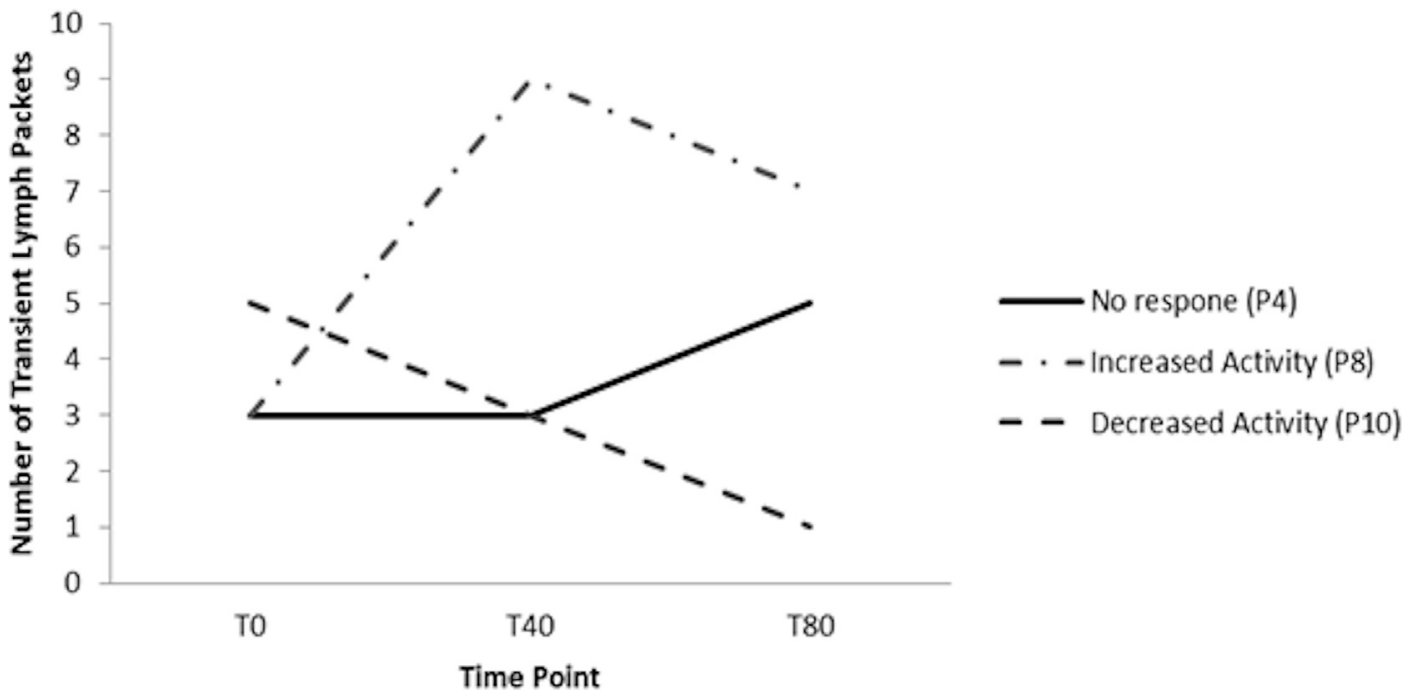

Fig. 9. Exemplars of three responses to mechanical loading over the secondary lymphatic vessels. 
pressure ulcers [6]. In addition, knowledge of the lymphatic architecture would enhance the understanding of lymph packet volume. Fluorescence imaging using ICG alone does not provide this information but when combined with magnetic resonance lymphography, there may be a basis for more accurate estimate of transient lymph packet size and volume [14].

Much research supports the view that dermal capillaries can collapse under clinically relevant magnitudes of uniaxial pressure (between 40 and $80 \mathrm{mmHg}$ ), leading to partial or total localised ischaemia $[15,16]$. The present study has shown that similar pressures can elicit changes in lymphatic function which might contribute to the cascade of mechanically-induced tissue damage, commonly leading to pressure ulcers. It has long been recognised that pressure ulcer aetiology may subsume a number of causative pathways, whose influence is dependent on both magnitude and time of mechanical loading [7]. The present study provides some insight into the potential mechanism of impaired fluid transport in loaded lymphatic vessels. Since reabsorption drives the flow of fluids through tissues, failure of the lymphatic system can result an accumulation of waste products, which can lead to cell death. The present study has shown that the velocity in which lymphatic packets travel up the limb reduces immediately post-loading and takes at least 40 mins to be restored to basal (T0) values (Tables 2 and 3). It is interesting to note that a reduction in velocity was also reported in the arms of lymphoedema patients with breast cancer, the magnitude of which increased in severe stages of the pathology [13]. More research is clearly needed to establish the physiological effects of these velocity changes and explore the impact of therapies such as exercise and manual lymph drainage techniques on impaired lymph vessels, particularly in those individuals at risk of lymphoedema and pressure ulcers.

\section{Conclusion}

The present study describes a methodology for assessing the effects of uniaxial mechanical loading on dermal lymphatic vessels in humans. A standardised protocol for data capture using ICG fluorescence imaging and analytical processing techniques were identified, which produced high levels of sensitivity and specificity when measuring transient lymphatic activity. Mechanical loading was shown to impair a number of quantifiable parameters reflecting lymphatic function in a proportion of the participants in the study, with significant changes in transient lymph packet velocity. Further research is required to define how lymphatic function is affected by pressure and shear forces.

\section{Conflicts of interest}

There were no conflicts of interest in this study.

\section{Funding}

The work was supported by the EPSRC-NIHR "Medical Device and Vulnerable Skin Network" (ref EP/M000303/1).

The University of Southampton funded a PhD studentship for Robert Gray.

\section{Ethical approval}

Ethics Approval for the study was granted by the Local Institutional Committee at the University of Southampton (REC ID: 8209).

\section{Acknowledgements}

The authors are grateful to FluoOptics (Grenoble, France) for their loan of the Fluobeam ${ }^{\circledR} 800$ system. We would also like to thank all of the participants who volunteered to participate in the study.

\section{Supplementary materials}

Supplementary material associated with this article can be found, in the online version, at doi:10.1016/j.medengphy.2016.04. 020 .

\section{References}

[1] Mortimer PS, Rockson SG. New developments in clinical aspects of lymphatic disease. J Clin Investig 2014;124:915-21.

[2] Stacker SA, Achen MG, Jussila L, Baldwin ME, Alitalo K. Metastasis: lymphangiogenesis and cancer metastasis. Nat Rev Cancer 2002;2:573-83.

[3] Paskett ED, Dean JA, Oliveri JM, Harrop JP. Cancer-related lymphedema risk factors, diagnosis, treatment, and impact: a review. J Clin Oncol. 2012;30:3726-33.

[4] Williams W, Witte C, Witte M, McNeill G. Radionuclide lymphangioscintigraphy in the evaluation of peripheral lymphedema. Clin Nucl Med. 2000;25:451-64.

[5] Sevick-Muraca EM. Translation of near-infrared fluorescence imaging technologies: emerging clinical applications. Ann Rev Med. 2012;63:217-31.

[6] Jung S-Y, Kim S-K, Kim S, Kwon Y, Lee E, Kang H-S, et al, Comparison of sentinel lymph node biopsy guided by the multimodal method of Indocyanine Green fluorescence, radioisotope, and Blue Dye versus the radioisotope method in breast cancer: a randomized controlled trial. Ann Surg Oncol 2014;21:1254-9.

[7] Bouten CVC, Oomens CWJ, Baaijens FPT, Bader DL. The aetiology of pressure ulcers: skin deep or muscle bound? Arch Phys Med Rehabil. 2003;84:616-19.

[8] Miller GE, Seale JL. Lymphatic clearance during compressive loading. Lymphology $1981 ; 14: 161-6$.

[9] Miller GE, Seale JL. The recovery of terminal lymph flow following occlusion. J Biomech Eng. 1987;109:48-54.

[10] Unno N, Nishiyama M, Suzuki M, Tanaka H, Yamamoto N, Sagara D, et al. A novel method of measuring human lymphatic pumping using indocyanine green fluorescence lymphography. J Vasc Surg. 2010;52:946-52.

[11] Gray RJ, Voegeli D, Bader DL. Features of lymphatic dysfunction in compressed skin tissues - Implications in pressure ulcer aetiology. J Tissue Viability 2016;25(1):26-31.

[12] Basu A. Droplet morphometry and velocimetry (DMV): a video processing software for time-resolved, label-free tracking of droplet parameters. Lab Chip 2013;13:1892-901.

[13] Yamamoto T, Narushima M, Yoshimatsu H, Yamamoto N, Kikuchi K, Todokoro T, et al. Dynamic Indocyanine Green (ICG) lymphography for breast cancer-related arm lymphedema. Ann Plast Surg. 2014;73:706-9.

[14] Lu Q, Delproposto Z, Hu A, Tran C, Liu N, Li Y, et al. MR Lymphography of lymphatic vessels in lower extremity with gynecologic oncology-related lymphedema. PLoS ONE 2012; 7:e50319.

[15] Wong VK, Stotts NA, Hopf HW, Froelicher ES, Dowling GA. How heel oxygenation changes under pressure. Wound Repair Regen. 2007;15:786-94.

[16] Chai CY, Bader DL. The physiological response of skin tissues to alternating support pressures in able-bodied subjects. J Mech Behav Biomed Mater 2013;28:427-35. 